\title{
Fabrication of 3D-Printed Ceramic Structures for Portable Solar
}

\section{Desalination Devices}

Win Jonhson, ${ }^{\text {a }}$ Xi Xu, ${ }^{1 \mathrm{a}, \mathrm{b}}$ Danwei Zhang,,${ }^{\ddagger \mathrm{a}}$ Wei Ting Chua, ${ }^{\mathrm{a}}$ Yong Hao Tan, ${ }^{\mathrm{a}}$ Ximeng Liu, ${ }^{\mathrm{a}}$

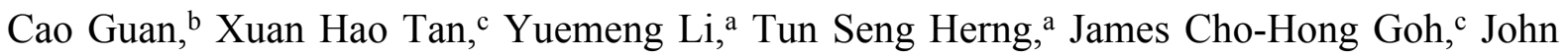
Wang, ${ }^{a}$ Hui He, ${ }^{* d}$ Jun Ding*a

a Department of Materials Science and Engineering Faculty of Engineering, National University of Singapore

9 Engineering Drive 1, Block EA, Singapore 117575

${ }^{b}$ Institute of Flexible Electronics, Xi'an Key Laboratory of Flexible Electronics, Northwestern Polytechnical University, Xi'an 710072, China.

c Department of Biomedical Engineering

Faculty of Engineering, National University of Singapore

4 Engineering Drive 3, Block E4, Singapore 117583

d School of Mechanical Engineering, F306 Mechanical Building 800 Dongchuan Road Minhang, Shanghai Jiaotong University, Shanghai 200240, China

*Corresponding authors E-mail: ME_hehui@sjtu.edu.cn (H. He), msedingj@,nus.edu.sg(J. Ding)

${ }^{1}$ These authors contributed equally: Win Jonhson, Xi Xu, and Danwei Zhang. 


\section{S1. Robocasting of Ceramic Solar Desalination Device}

Fabrication of the fully ceramic solar desalination device was done by using Regenhu 3D

Discovery (Switzerland). A single printhead with nozzles of size $410 \mu \mathrm{m}$ was used. There is no specific requirement for the substrate used in the printing process. In this paper, a Polyethylene terephthalate (PET) film was used as the film substrate. Figure S1 shows the robocasting setup used for the fabrication process.

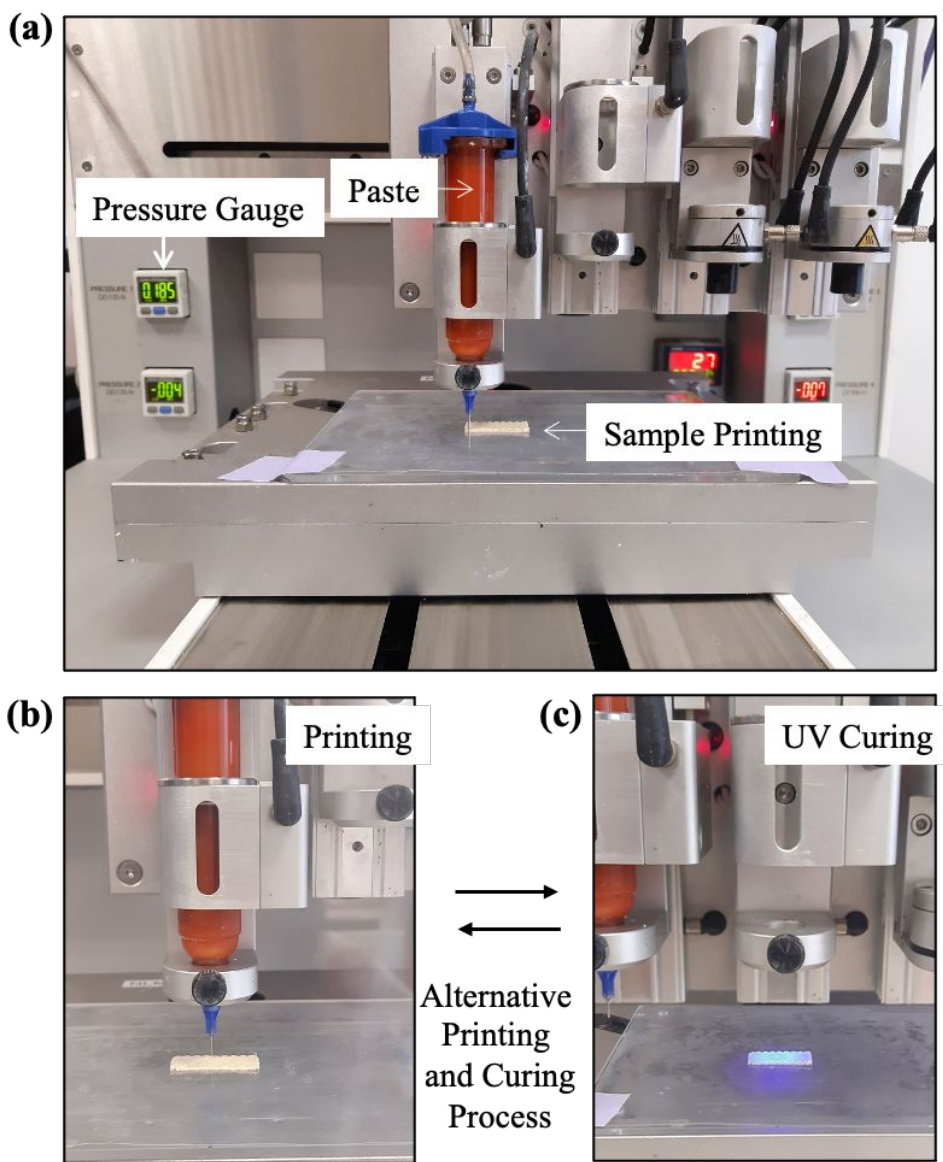

Figure S1. a) Overview of the robocasting setup. b) Portable solar desalination device printing in progress using the formulated silica paste. c) The alternating UV curing in progress. 


\section{S2. Wicking Study of Cotton and Silica}

Wicking is a natural phenomenon that is driven by capillary forces. The wicking height depends on many factors, one of which is the capillary diameter. Currently, the research of ceramic wick is limited, and it has not been used for desalination purposes to the best of our knowledge.

A study was conducted to find the fastest and most efficient water uptake of cotton. Different densities of cotton were prepared by differing the mass while maintaining the volume of 4.05 $\mathrm{ml}(1.5 \mathrm{~cm} \times 1.5 \mathrm{~cm} \times 1.8 \mathrm{~cm})$. The differences in height were recorded at different timing and are shown as follows:

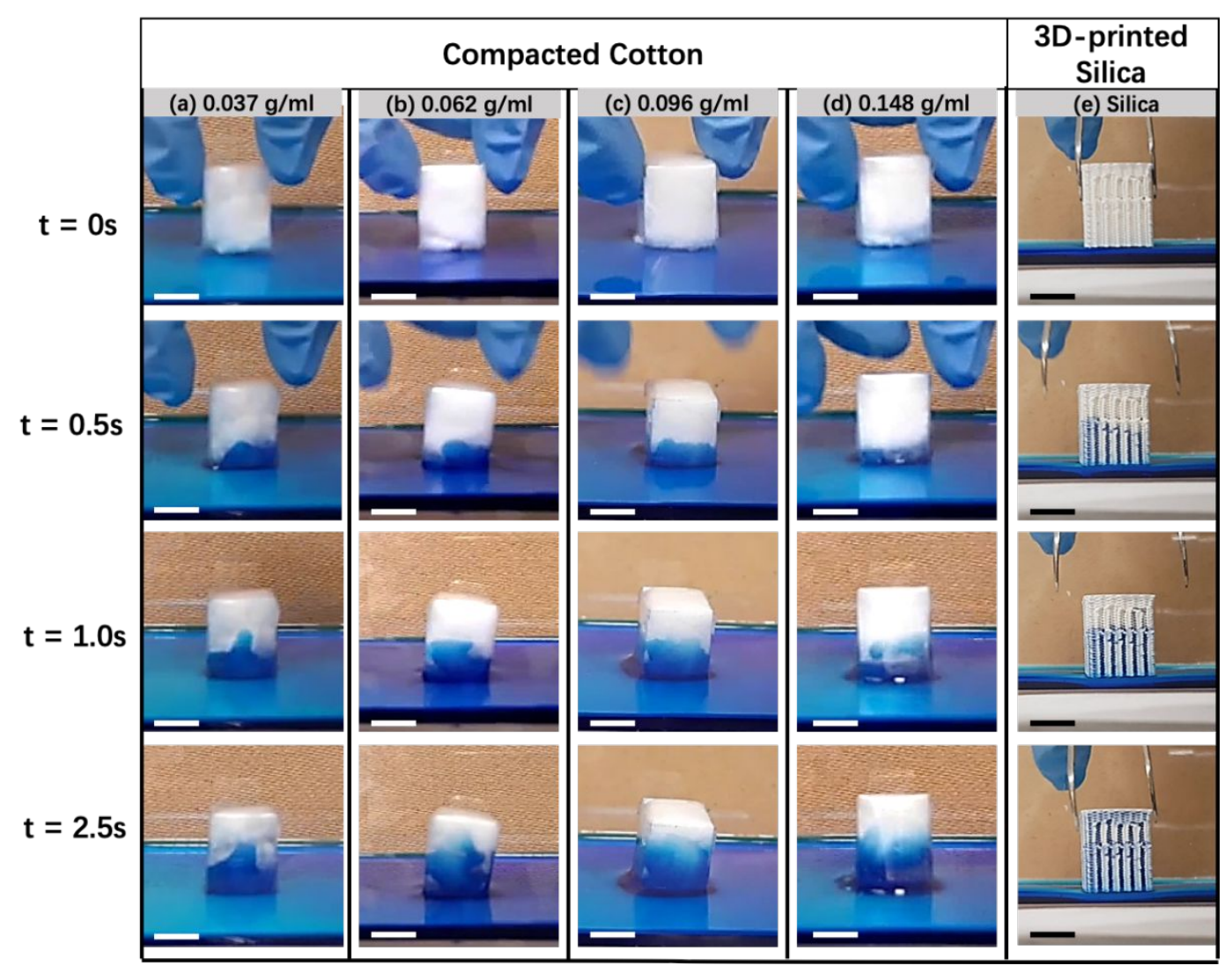

Figure S2. Wicking study of (a-d) compacted cotton with different density and e) 3D-printed silica of density $2.65 \mathrm{~g} / \mathrm{ml}$. All scale bar is equivalent to $1 \mathrm{~cm}$. 

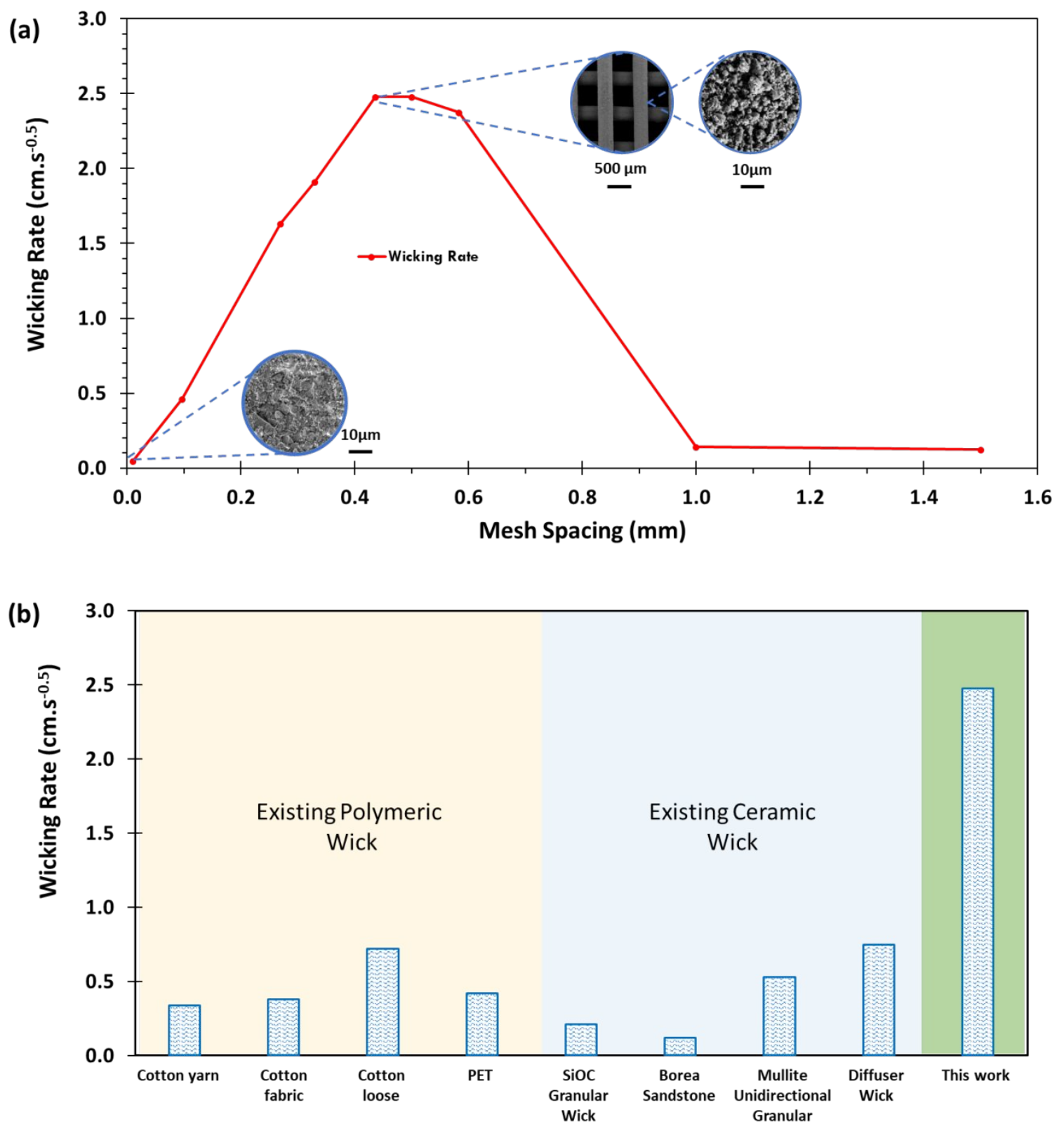

Figure S3. a) Wicking rate of silica wick with different mesh spacing. b) Wicking performance comparison of Polymeric Wick: Cotton yarn ${ }^{1}$, cotton fabric ${ }^{1}$, experimented compacted loose cotton (see also Figure S2), and PET ${ }^{1}$, Ceramic Wick: SiOC ${ }^{2}$, Borea Sandstone $^{3}$, Mullite ${ }^{4}$, Diffuser (Different Ceramic Matrix) ${ }^{5}$ and proposed wick. 


\section{S3. Sintering Study}

The post-printing heat treatment process was necessary to obtain pure ceramic structures without polymer content. To allow complete plasticizer burn-off, the structures were subjected to thermal heating at $150^{\circ} \mathrm{C}$ for several hours. Debinding was done at temperatures between $400^{\circ} \mathrm{C}$ to $600^{\circ} \mathrm{C}$ based on the thermogravimetric analysis in main Figure $2 \mathrm{f}$. A systematic sintering study was performed to find the optimum nano-pores to suit the application. Sintered samples of different sintering temperatures were prepared to be observed under a scanning electron microscope (SEM) by using Zeiss; FESEM Supra 40 with $5 \mathrm{kV}$ acceleration voltage without additional sample preparation, e.g., polishing, grinding, or gold sputtering. Figure below shows the grain growth of the sintered sample at $1200^{\circ} \mathrm{C}, 1300^{\circ} \mathrm{C}$, and $1400^{\circ} \mathrm{C}$.
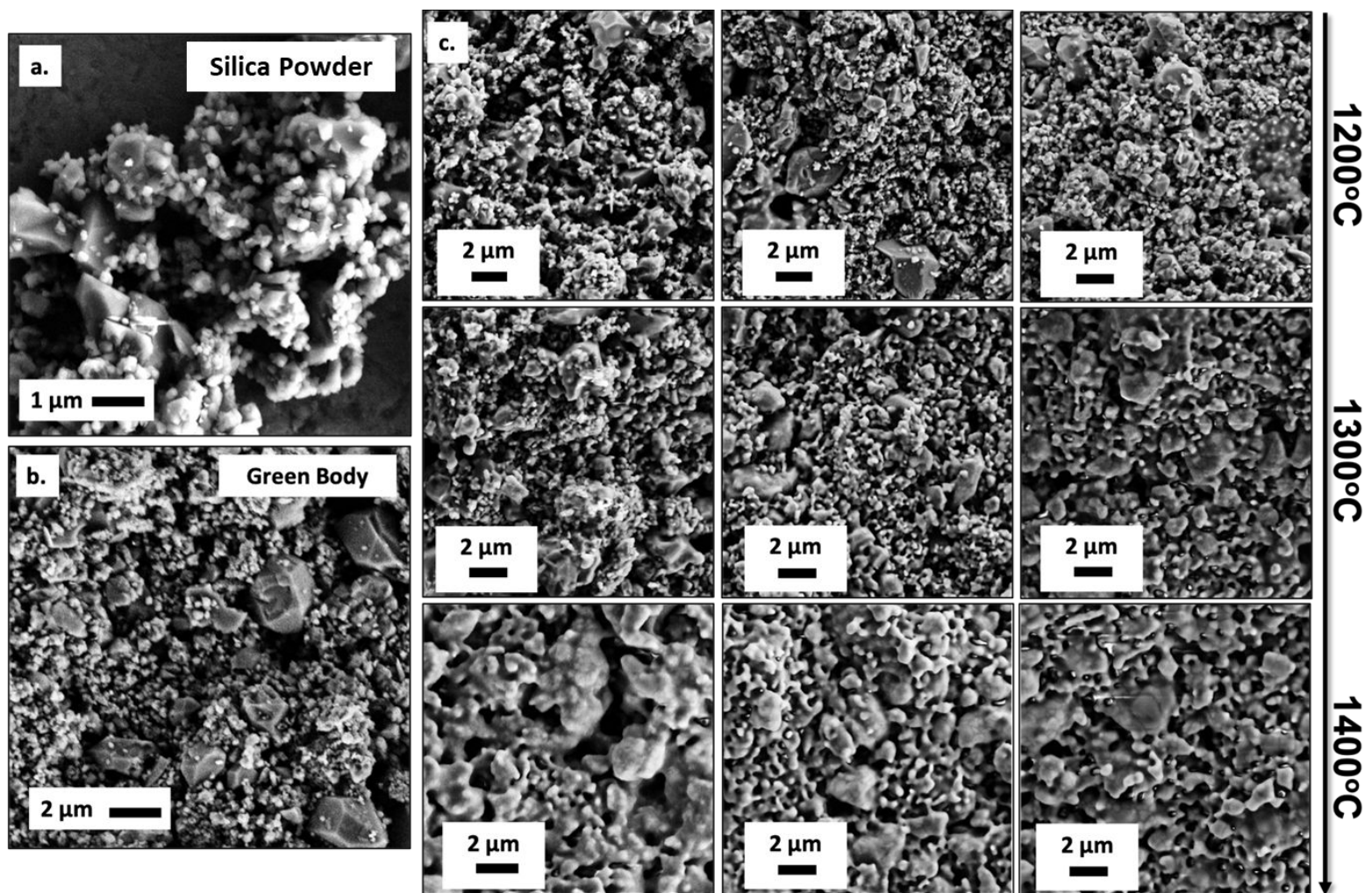

$2 \mu \mathrm{m}$

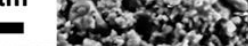

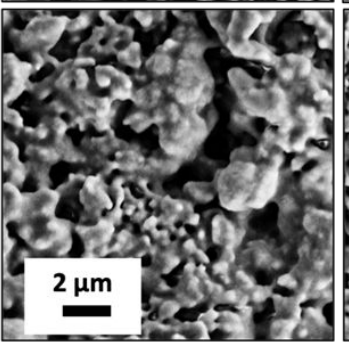

2 Hours
5 Hours

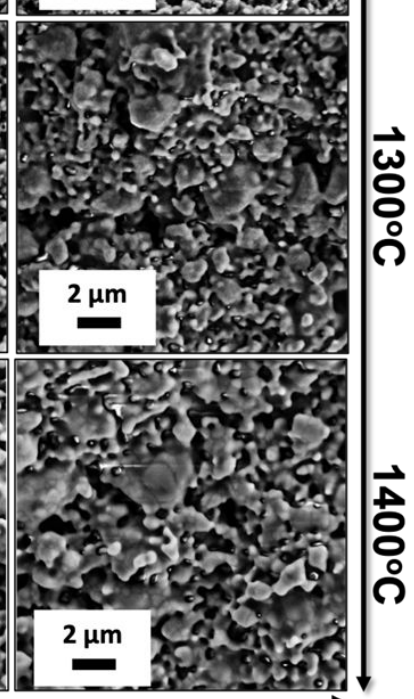

10 Hours

Figure S4. Images of a) starting powder; b) silica green-body after debinding, evaporation of plasticizer; c) sintered silica with different sintering temperature $\left(1200^{\circ} \mathrm{C}, 1300^{\circ} \mathrm{C}\right.$ and $\left.1400^{\circ} \mathrm{C}\right)$ and annealing time ( 2 hours, 5 hours and 10 hours) accordingly. 


\section{S4. Water absorption and shrinkage study}

A water contact angle measurement equipment, VCA Optima by AST Product, was used to measure the absorption time of $5 \mathrm{~mL}$ water on different porous silica structures. Figure S5a shows that silica sintered at $1400^{\circ} \mathrm{C}$ has the shortest absorption time compared to silica sintered at $1200^{\circ} \mathrm{C}$ and $1300^{\circ} \mathrm{C}$. The absorption time does not differ significantly between silica sintered at $1400^{\circ} \mathrm{C}$ with different annealing times. Hence, 2 hours of annealing time was selected to be the sintering parameter.

(a)

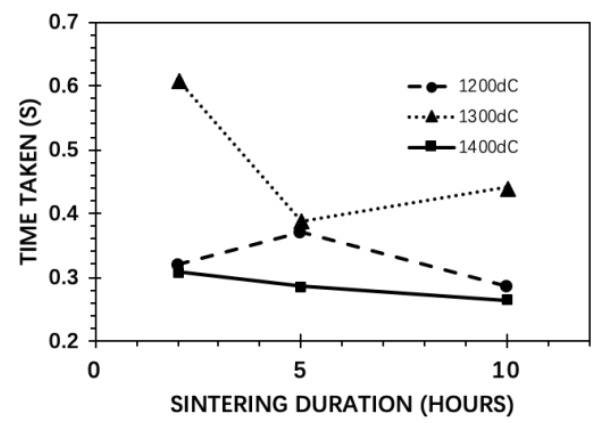

(b)

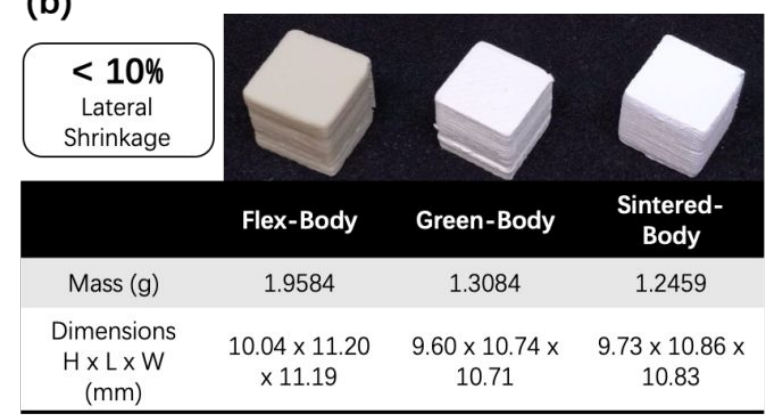

Figure S5. a) Time taken for $5 \mu \mathrm{L}$ water to be absorbed on the surface with different sintering duration. b) Lateral shrinkage during the debinding and sintering.

Shrinkage study was performed by comparing the mass and dimension of freshly printed structure (flex-body), debinded structure (green-body) and, sintered body. Figure S5b shows that the lateral shrinkage of the sintered silica is less than $10 \%$ of the flex-body. This minimal shrinkage is needed to produce a high-quality porous solar absorber and wick. 
S5. Mesh Spacing SEM Images

(a)

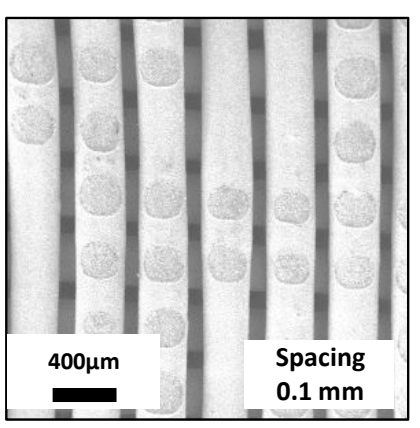

(d)

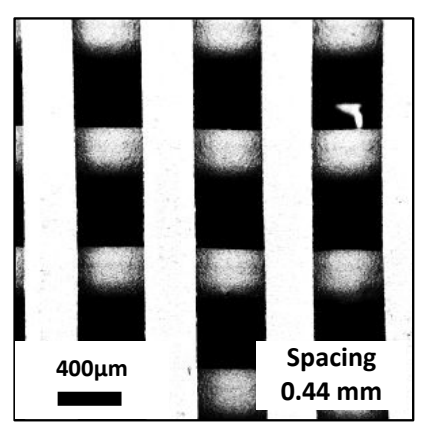

(b)

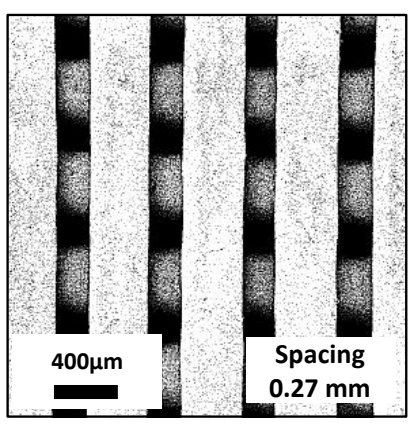

(e)

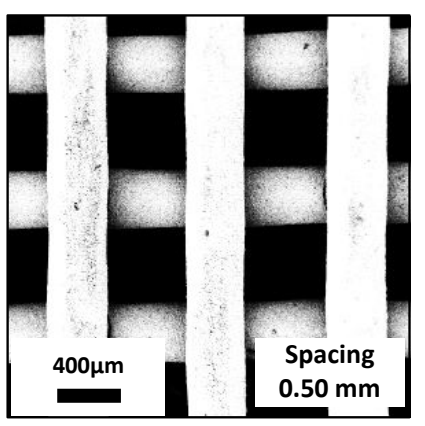

(c)

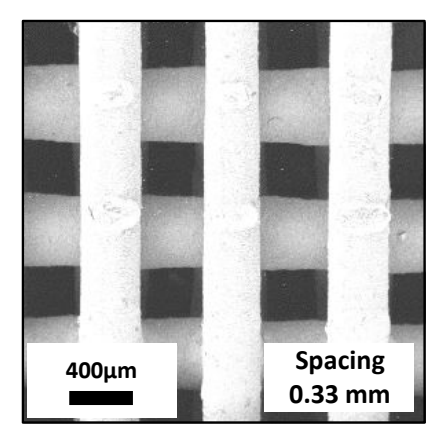

(f)

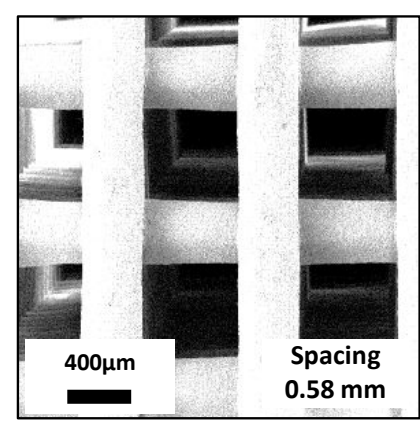

Figure S6. (a-f) SEM images of printed silica mesh structures with different pore area. 


\section{S6. Surface Functionalization}

The ceramic floater was prepared by hydrolytic deposition of fluoroalkylsilane on the silica substrate. ${ }^{6-7}$ Multiple planar layers acting as microfibrillar were formed with superoleophobic and superhydrophobic properties. ${ }^{8}$ The structure was washed thoroughly in ethanol to achieve the desired properties without any contamination before any surface treatment. For a strong chemical bond of TEHDFS onto the silica backbone, the silica structure is first etched in $\mathrm{KOH}$ to increase surface roughness and become more reactive (dangling bond of $\mathrm{Si}$ ) ${ }^{9}$. The formation of silane monolayers ${ }^{6-7}$ on surfaces exposing free hydroxyl groups has been experimentally proven $^{7}$. Finally, the structure was immersed in TEHDFS/ethanol solution by stirring at 120 rpm on a table roller for 24 hours. In Figure S7b and S7c, the ceramic floater showed apparent water-in-air and oil-in-water contact angles of more than $150^{\circ}$, indicating the superhydrophobic and superoleophobic properties of TEHDFS coating on silica.
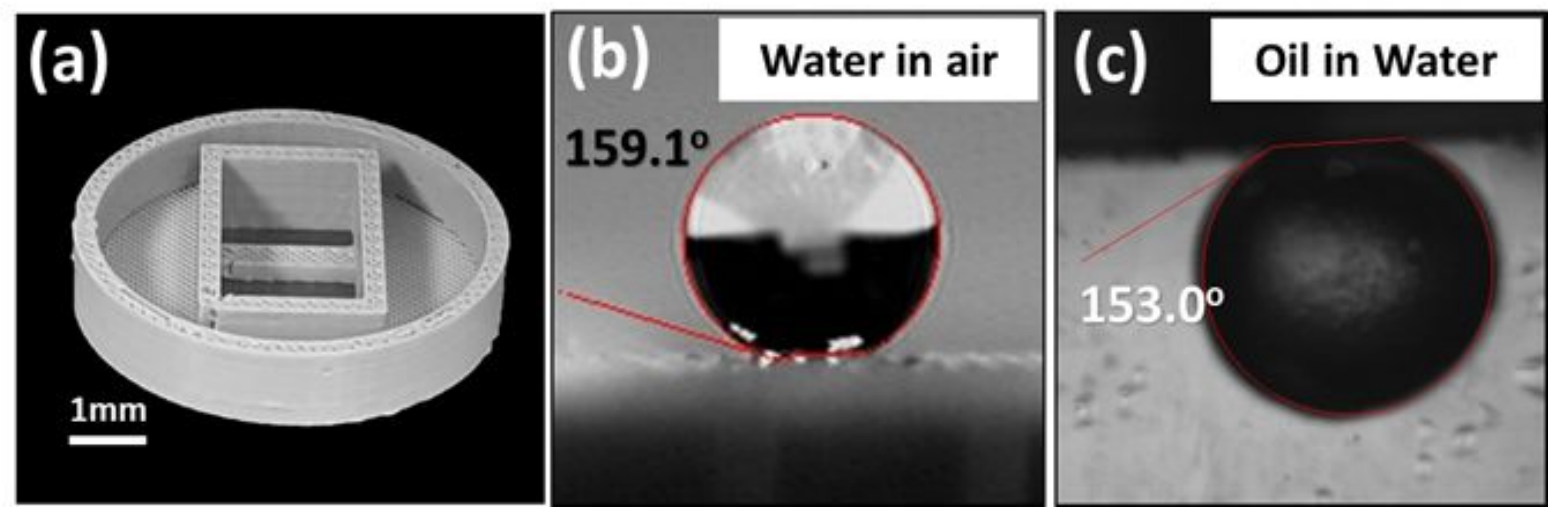

Figure S7. a) TEHDFS coated ceramic floater (initial design). b) Water contact angle measurement of TEHDFS. c) Oil-in-water contact angle measurement of TEHDFS.

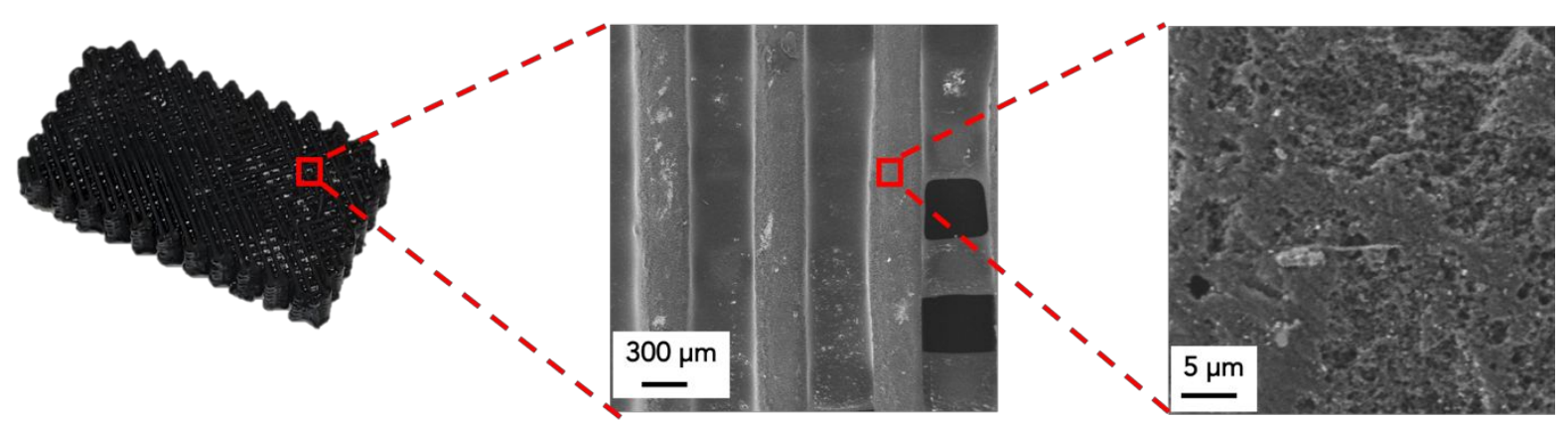

Figure S8. Surface coated silica mesh with black aerosol paint. 


\section{S7. Thermal Conductivity}

The thermal conductivity of MG@silica and silica wick were measured using Fourier's Law for heat conduction as follows:

$$
\dot{Q}=-k A \frac{\Delta T}{L}
$$

Where $\dot{Q}$ is the solar flux, $k$ is the thermal conductivity, $A$ is the plane area exposed to solar flux, $\Delta T$ is the temperature difference from top and bottom surface of the samples, and $L$ is the distance from top to bottom surface.

As shown in Figure S9, an experiment was conducted to measure the thermal conductivity of MG@silica and silica wick. A set of thin black-painted aluminum plates was placed on top and bottom of the sample. The samples were exposed to solar irradiance of $1000 \mathrm{~W} . \mathrm{m}^{-2}$ (corresponding to AM1.5G) for 10 mins to achieve thermal equilibrium (temperature variation was close to $0^{\circ} \mathrm{C}$ ). The samples have an identical thickness of $0.5 \mathrm{~cm}$.

(a)

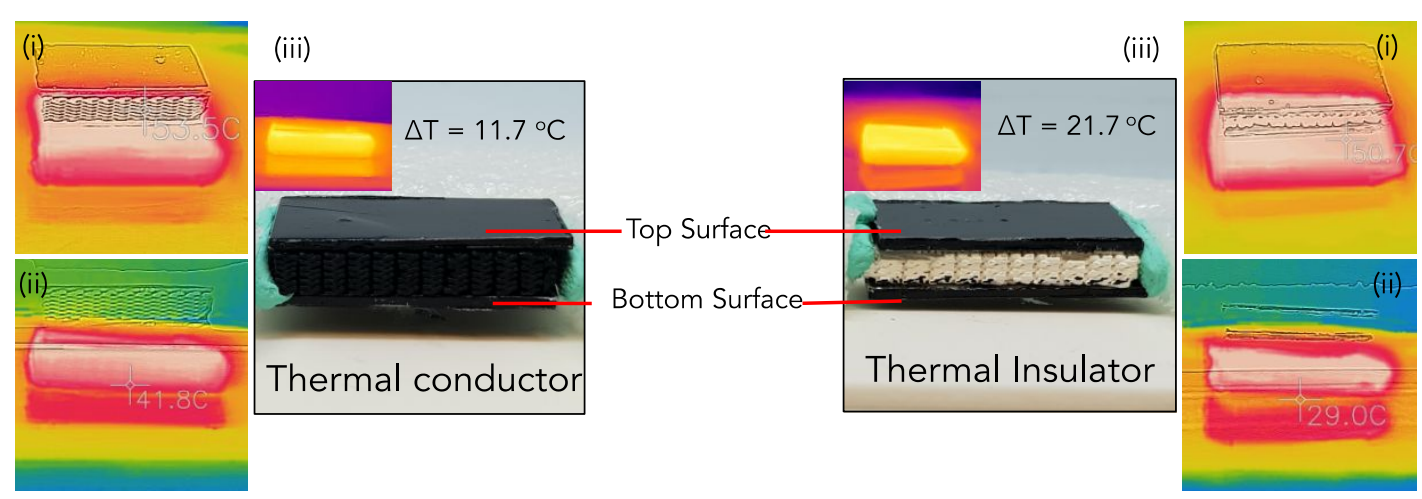

(b)

Figure S9. Thermal Conductivity of MG@Silica and Silica. a) Modified graphene oxide on silica (MG@silica) (i) top surface temperature=53.5 $5^{\circ}$; (ii) bottom surface temperature = $41.8^{\circ} \mathrm{C}$; (iii) digital image of the setup. b) Silica wick (i) top surface temperature $=50.7^{\circ} \mathrm{C}$; (ii) bottom surface temperature $=29.0^{\circ} \mathrm{C}$; (iii) digital image of the setup.

From the equation above. It can be calculated that MG@silica has a thermal conductivity of $0.43 \mathrm{~W} \cdot \mathrm{m}^{-1} \cdot \mathrm{K}^{-1}$ while silica wick has a thermal conductivity of $0.23 \mathrm{~W} \cdot \mathrm{m}^{-1} \cdot \mathrm{K}^{-1}$. 


\section{S8. Solar Steam Generation}

The solar absorber material was fabricated using chemical vapor deposition of graphene on the as-fabricated silica mesh surface. ${ }^{10}$ To obtain a hydrophilic surface which is more favorable in a floating system, the graphene-coated silica mesh was immersed in $65 \%$ nitric acid $\left(\mathrm{HNO}_{3}\right)$ aqueous solution. Solar efficiency and steam generation experiments were conducted using Newport 92250A-1000 Watts solar simulator with Newport 91150 PVM191 as a reference cell meter. All experiments were conducted with a spectral irradiance standard of AM 1.5G. The solar steam generation efficiency was tested in the punched polystyrene foam (thermal conductivity $\left.\approx 0.04 \mathrm{~W} \cdot \mathrm{m}^{-1} \cdot \mathrm{K}^{-1}\right)$ with the MG@Silica inserted in the opening. The entire structure floats on the water, with only a part of the ceramic wick $(\sim 0.5 \mathrm{~cm})$ was in contact with the water. The experiments were conducted at an ambient temperature of $\sim 20^{\circ} \mathrm{C}$ and a humidity of $\sim 41 \%$ in a beaker glass. The efficiency of solar-to-vapor conversion is calculated as follows: ${ }^{11-13}$

$$
\begin{gathered}
\eta=\frac{m\left(L_{v}+Q\right)}{P_{\text {in }}} \\
L_{v}=1.91846 \times 10^{6}\left(\frac{T}{T-33.91}\right)^{2} \\
Q=c\left(T-T_{1}\right)
\end{gathered}
$$

Where $\mathrm{m}$ is the rate of mass loss of water under illumination (after subtracting dark evaporation), $\mathrm{L}_{\mathrm{v}}$ is the total latent heat of vaporization of water from liquid to vapor phase in the region of interest, $\mathrm{Q}$ is the energy provided to heat the system from the initial temperature $\mathrm{T}_{1}$ to a average steady temperature of $\mathrm{T}$, and $\mathrm{P}_{\text {in }}$ is the incident light power on the solar absorber. The measurements for the enhanced 3D design of MG@silica / silica mesh were conducted three times, with each cycle lasting for 24 hours under the solar simulator. 

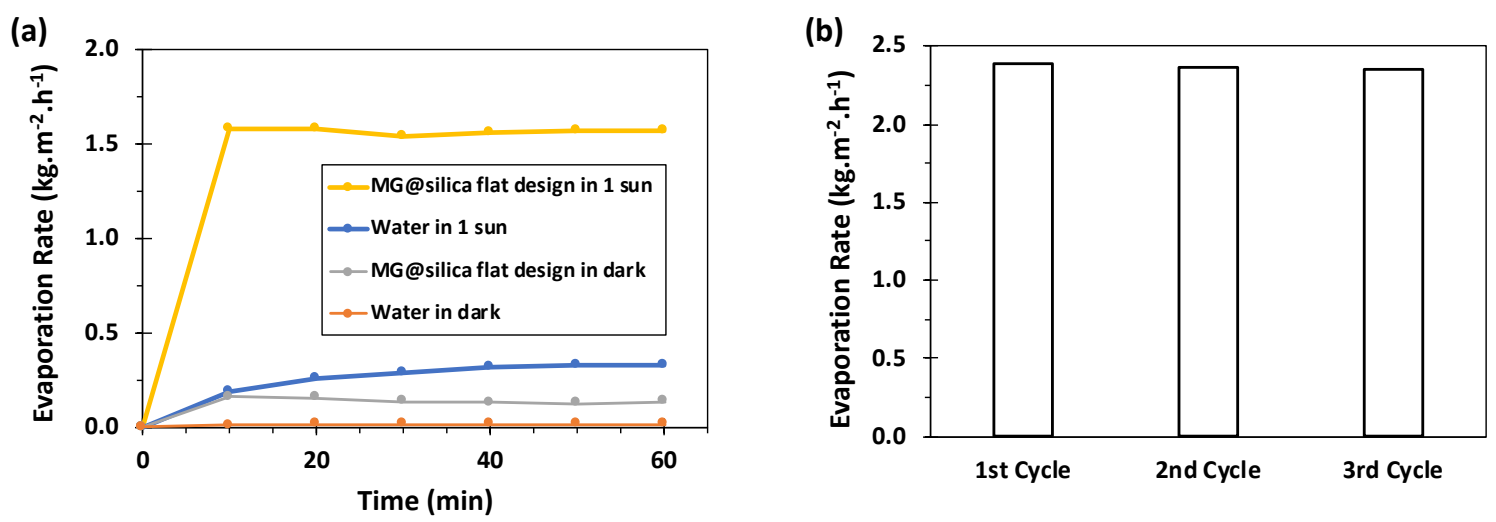

Figure S10. (a) Evaporation rate of MG@silica flat design and bulk water in dark and 1 sun. (b) Average evaporation rate of enhanced 3D design after 1st, 2nd and, 3rd cycling test of 24 hours for each test. 
S9. Mechanical Strength

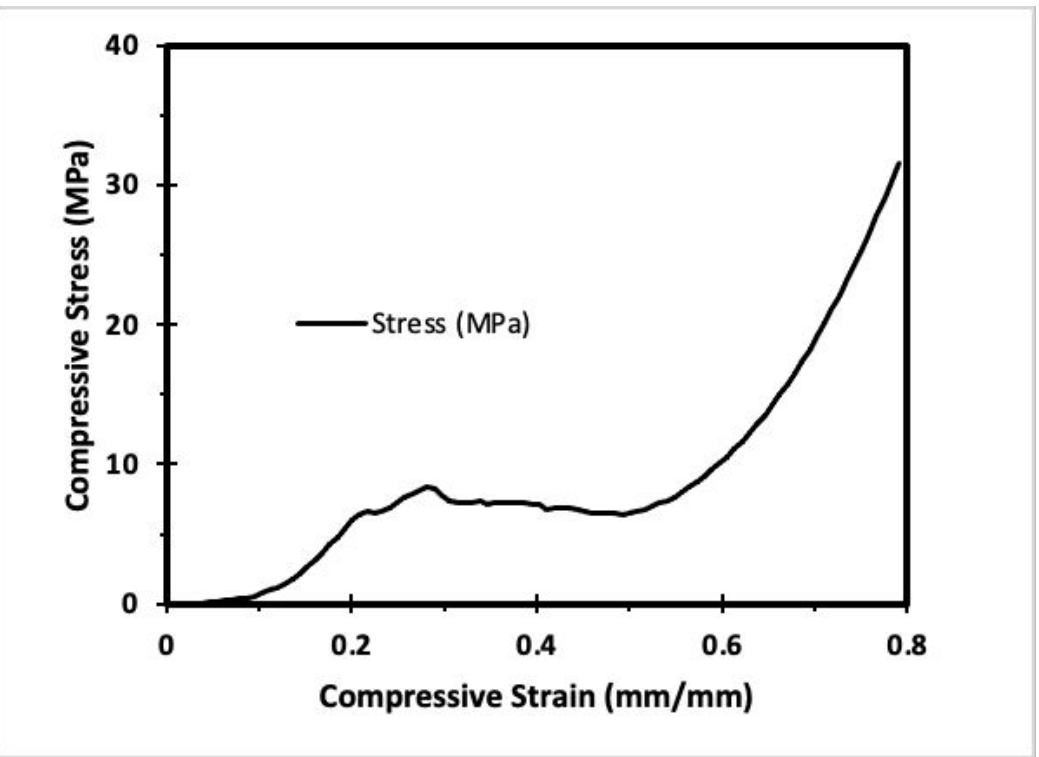

Figure S11. Compressive stress-strain curve of 3D printed silica mesh. The test sample were deformed up to $80 \%$ strain without cracking. 


\section{S10. Microbiomes Study}

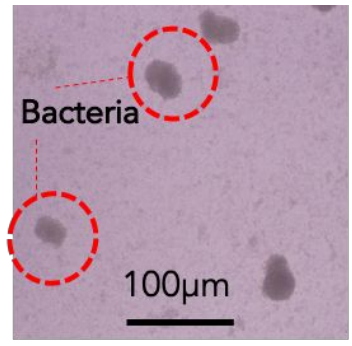

Seawater

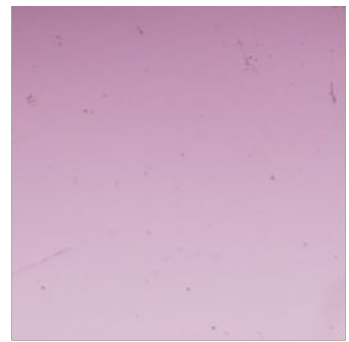

Desalinated

water

Figure S12. Microscopic image of seawater and desalinated water.

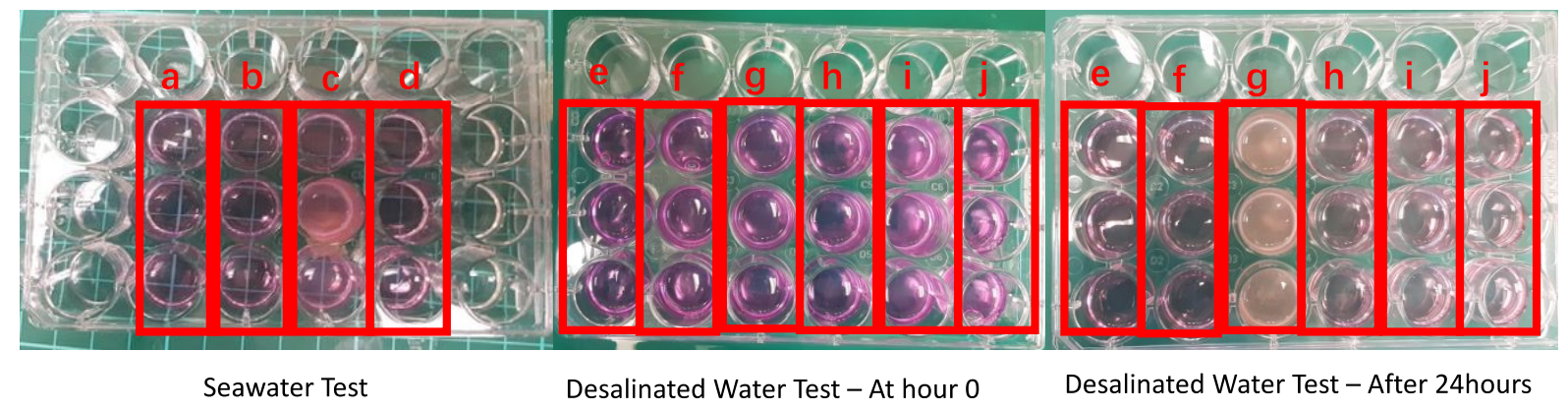

Figure S13. Bacteria test on Seawater and Desalinated Water. a) Negative control without antibiotics; b) negative control with antibiotics; c) positive control without antibiotics; d) positive control with antibiotics; e) same as a); f) same as b); g) same as c); h) same as d); i) test sample without antibiotics; j) test sample with antibiotics.

The presence of bacterial microbes was tested by performing cell culture in a culture medium. Briefly, the culture medium was made using high glucose DMEM with Glutamax, supplemented with 10\% Fetal Bovine Serum (FBS). Samples tested with antibiotics had their culture mediums further supplemented with 1000ng/ml Penicillin and $100 \mathrm{ng} / \mathrm{ml} \mathrm{Gentamycin.}$ Culture medium with or without antibiotics was then added to a $24-w e l l$ TCP plate. For negative controls, only $500 \mu \mathrm{L}$ of culture medium (with or without antibiotics) was added to well plates. For positive controls, $500 \mu \mathrm{L}$ of culture medium (with or without antibiotics) was added to the well plates first, to which $50 \mu \mathrm{L}$ of seawater was added. For desalinated water, $500 \mu \mathrm{L}$ of culture medium (with or without antibiotics) was added to the well plates first, to which $50 \mu \mathrm{L}$ of desalinated water was added. The well plate was then cultured in $37^{\circ} \mathrm{C}$ and $5 \%$ $\mathrm{CO}_{2}$ for 24 hours before observing any bacterial presence. All items used were obtained from ThermoFisher Scientific. The desalinated water sample had neither observable clumped particulates nor observable moving particles (Figure S12). The seawater was observed to turn 
cloudy with media color changing to yellow in the absence of antibiotics but was translucent with no media color change when antibiotics were present (Figure S13g-h). For the desalinated water, no observable visual change regardless of the presence of antibiotics (Figure S13i-j). Taken together, these results indicate that the proposed desalination device is effective in removing bacterial microbes from the seawater. 


\section{S11. Cost Analysis}

The portable desalination device was designed to be fully ceramic by using inexpensive silica and non-toxic chemical for $3 \mathrm{D}$ printing paste. The material cost is summarized as below:

- $50 \mathrm{lbs}$ of silica powder was priced around SGD 774 from Sigma Aldrich, Singapore. $5.3 \mathrm{~g}$ of silica was used for this prototype model. Cost: SGD 0.19.

- EG was purchased from Sigma Aldrich, Singapore at SGD 188 for 1 L. 1.4 ml was used to make the paste. Cost: SGD 0.26 .

- PEGDA was purchased from Sigma Aldrich, Singapore at SGD 200 for $500 \mathrm{ml} .1 .5 \mathrm{ml}$ was used to make the paste. Cost: SGD 0.60

- TPO was purchased from TCI Chemical at SGD 88.35 for $25 \mathrm{~g} .17 \mathrm{mg}$ was used to make the paste. Cost: SGD 0.06

The total material cost to make the 3D printing paste was SGD 1.11 (less than USD 1) for a prototype of $5 \mathrm{~cm}^{2}$. Scaling up can further reduce the cost by purchasing the materials at bulk quantities. The device can produce $\sim 0.5 \mathrm{~L}$ of water per $\mathrm{m}^{2}$ within an hour and with the assumption of 10 hours of sun per day (in South East Asia), the device can produce an average of $5 \mathrm{~L}$ of water per $\mathrm{m}^{2}$ per day. The device itself has no lifetime limit due to the ceramic core structure hence the unlimited fresh water supply for a very long time. 


\section{References}

(1) Mhetre, S. K. Effect of Fabric Structure on Liquid Transport, Ink Jet Drop Spreading and Printing Quality. Georgia Institute of Technology, 2009.

(2) Grebenyuk, Y.; Zhang, H.; Wilhelm, M.; Rezwan, K.; Dreyer, M. Wicking into Porous Polymer-Derived Ceramic Monoliths Fabricated by Freeze-Casting. Journal of the European Ceramic Society 2017, 37 (5), 1993-2000.

(3) Tsunazawa, Y.; Yokoyama, T.; Nishiyama, N. An Experimental Study on the Rate and Mechanism of Capillary Rise in Sandstone. Progress in Earth and Planetary Science 2016, 3 (1), 8.

(4) Popa, C.; Okayasu, Y.; Katsumata, K.-i.; Isobe, T.; Matsushita, N.; Nakajima, A.; Kurata, T.; Okada, K. Capillary Rise Properties of Porous Mullite Ceramics Prepared by an Extrusion Method with Various Diameters of Fiber Pore Formers. Journal of Materials Science 2013, 48 (2), 941-947.

(5) Pisklak, T. J., Fragrance Dispensing Wick and Method. Google Patents: 2013.

(6) Untereker, D.; Lennox, J. C.; Wier, L.; Moses, P.; Murray, R. W. Chemically Modified Electrodes: Part Iv. Evidence for Formation of Monolayers of Bonded Organosilane Reagents. Journal of Electroanalytical Chemistry and Interfacial Electrochemistry 1977, 81 (2), 309-318.

(7) Sagiv, J. Organized Monolayers by Adsorption. 1. Formation and Structure of Oleophobic Mixed Monolayers on Solid Surfaces. Journal of the American Chemical Society 1980, 102 (1), 92-98, DOI: 10.1021/ja00521a016.

(8) Arkles, B. Hydrophobicity, Hydrophilicity and Silane Surface Modification. Gelest Inc, Morrisville 2011.

(9) Chandra, P.; Doke, D. S.; Umbarkar, S. B.; Vanka, K.; Biradar, A. V. Silica Microspheres Containing High Density Surface Hydroxyl Groups as Efficient Epoxidation Catalysts. RSC Advances 2015, 5 (27), 21125-21131.

(10) Xu, X.; Guan, C.; Xu, L.; Tan, Y. H.; Zhang, D.; Wang, Y.; Zhang, H.; Blackwood, D. J.; Wang, J.; Li, M. Three Dimensionally Free-Formable Graphene Foam with Designed Structures for Energy and Environmental Applications. ACS nano 2019, 14 (1), 937-947.

(11) Gao, M.; Zhu, L.; Peh, C. K.; Ho, G. W. Solar Absorber Material and System Designs for Photothermal Water Vaporization Towards Clean Water and Energy Production. Energy \& Environmental Science 2019, 12 (3), 841-864, DOI: 10.1039/C8EE01146J.

(12) Hu, T.; Li, L.; Yang, Y.; Zhang, J. A Yolk@Shell Superhydrophobic/Superhydrophilic Solar Evaporator for Efficient and Stable Desalination. Journal of Materials Chemistry A 2020, 8 (29), 14736-14745, DOI: 10.1039/DOTA04917D.

(13) Wu, X.; Robson, M. E.; Phelps, J. L.; Tan, J. S.; Shao, B.; Owens, G.; Xu, H. A Flexible Photothermal Cotton-Cus Nanocage-Agarose Aerogel Towards Portable Solar Steam Generation. Nano Energy 2019, 56, 708-715, DOI: https://doi.org/10.1016/j.nanoen.2018.12.008. 